\title{
Central nervous system systemic lupus erythematosus mimicking progressive multifocal leucoencephalopathy
}

\author{
Brian R Kaye, C Michael Neuwelt, Stuart S London, Stephen J DeArmond
}

\begin{abstract}
The case is reported of a patient with central nervous system systemic lupus erythematosus (SLE) with features of progressive multifocal leucoencephalopathy (PML) seen clinically and by magnetic resonance imaging. $A$ brain biopsy sample showed microinfarcts. The use of magnetic resonance imaging and IgG synthesis rates in evaluating central nervous system lupus, the co-occurrence of SLE and PML, and the differentiation of these entities by magnetic resonance imaging and by histology are considered.
\end{abstract}

(Ann Rheum Dis 1992; 51: 1152-1156)

The central nervous system manifestations of systemic lupus erythematosus (SLE) are protean. Strokes, seizures, acute psychosis, depression, focal neurological impairment, and subtle psychological abnormalities are among the myriad of neurological findings in patients with SLE. One finding in patients with SLE is progressive multifocal leucoencephalopathy (PML). ${ }^{1-5}$ Magnetic resonance imaging has been used for the ongoing evaluation of central nervous system lupus ${ }^{6-10}$ and PML, ${ }^{11}$ perhaps increasing our rate of identification of these two diseases when they occur concomitantly.

We report here the case of a patient with SLE who had neurological findings and magnetic resonance imaging abnormalities that mimicked PML. A brain biopsy sample was taken from the patient while she was still alive, providing pathological correlation with the magnetic resonance imaging scan. This case shows the need to use several different diagnostic techniques in evaluating central nervous system manifestations of SLE. It also reinforces the need to consider serious brain disease in patients with SLE showing subtle psychological abnormalities.

\section{Case report}

A 35 year old white female speech pathologist presented in December 1982 with one month's history of pleuritic chest pain and an erythematous urticarial rash following a laparotomy for a ruptured appendix. Two and a half months previously she had delivered a healthy infant. Her pregnancy had been marked by polyarthralgias. Physical examination revealed only a fine erythematous, urticarial rash on her chest, hands, and feet. Initial laboratory evaluation included a normal complete blood count, chemistry panel, and urine analysis. A test for antinuclear antibodies was positive at a 1:80 dilution. Antibodies to double stranded DNA were negative and serum complement concentrations were normal.

The patient did well until June 1983 when she again developed pleuritic chest pain and swelling of her hands. At this time an antinuclear antibody test was positive at a 1:320 dilution with a speckled pattern. Antibodies to ribonucleoprotein, Sm, SS-A, and SS-B were absent. Her complete blood count and urine analysis were normal.

In November 1985 she developed daily fevers, fatigue, and malar rash. Her packed cell volume was 0.34 and a direct Coombs' test was positive. A diagnosis of SLE was made and she was treated with hydroxychloroquine $(400 \mathrm{mg} /$ day $)$. One month later she had a brief psychotic reaction.

In February 1986 she developed mouth ulcers and erythema on her fingertips. She was noted to have slowing of her mentation. At that time her white blood cell count had decreased to $4 \cdot 4 \times 10^{6} / 1$, her packed cell volume was $0 \cdot 31$, and her erythrocyte sedimentation rate was $52 \mathrm{~mm} /$ hour (Westergren method). She had $3+$ blood on a urine dipstick test. She was treated with prednisone ( $10 \mathrm{mg} /$ day), which was subsequently increased to $20 \mathrm{mg} /$ day.

In March 1986, after a trip to Hawaii, she developed a fever to $40^{\circ} \mathrm{C}$, difficulty concentrating, dysarthria, and a tremor of her left hand. Antibodies to double stranded DNA, direct Coombs' test, and antibodies to extractable nuclear antigen were negative; total haemolytic complement concentrations were normal. An analysis of her cerebral spinal fluid gave a white blood cell count of $0.03 \times 10^{6} / 1$ with $91 \%$ lymphocytes, $7 \%$ monocytes, and $2 \%$ polymorphonuclear leucocytes, and a negative herpes simplex virus antibody test. The IgG synthesis rate in the cerebrospinal fluid was $0.46 \mathrm{~g} / 24$ hours (normal value less than 0.033 $\mathrm{g} / 24$ hours), cerebrospinal fluid protein $500 \mathrm{~g} / 1$ (normal less than $450 \mathrm{~g} / \mathrm{l}$ ), and cerebrospinal fluid glucose $4.1 \mathrm{mmol} / \mathrm{l}$. Her peripheral white blood cell count was $3 \cdot 1 \times 10^{6} / 1$ with normal serum $\mathrm{C} 3$ and $\mathrm{C} 4$ concentrations. An initial magnetic resonance imaging scan of her brain showed a confluent high signal intensity on proton density and $T_{2}$ weighted images within the centrum semiovale and the coronal radiata consistent with demyelination (fig 1). An electroencephalogram showed a mild, generalised, non-specific abnormality that was consistent \\ East Bay Rheur
Medical Group,
Oakland and \\ San Leandro, \\ Medicine, \\ B R Kaye \\ S S London \\ Pathology, \\ University of Calif
}



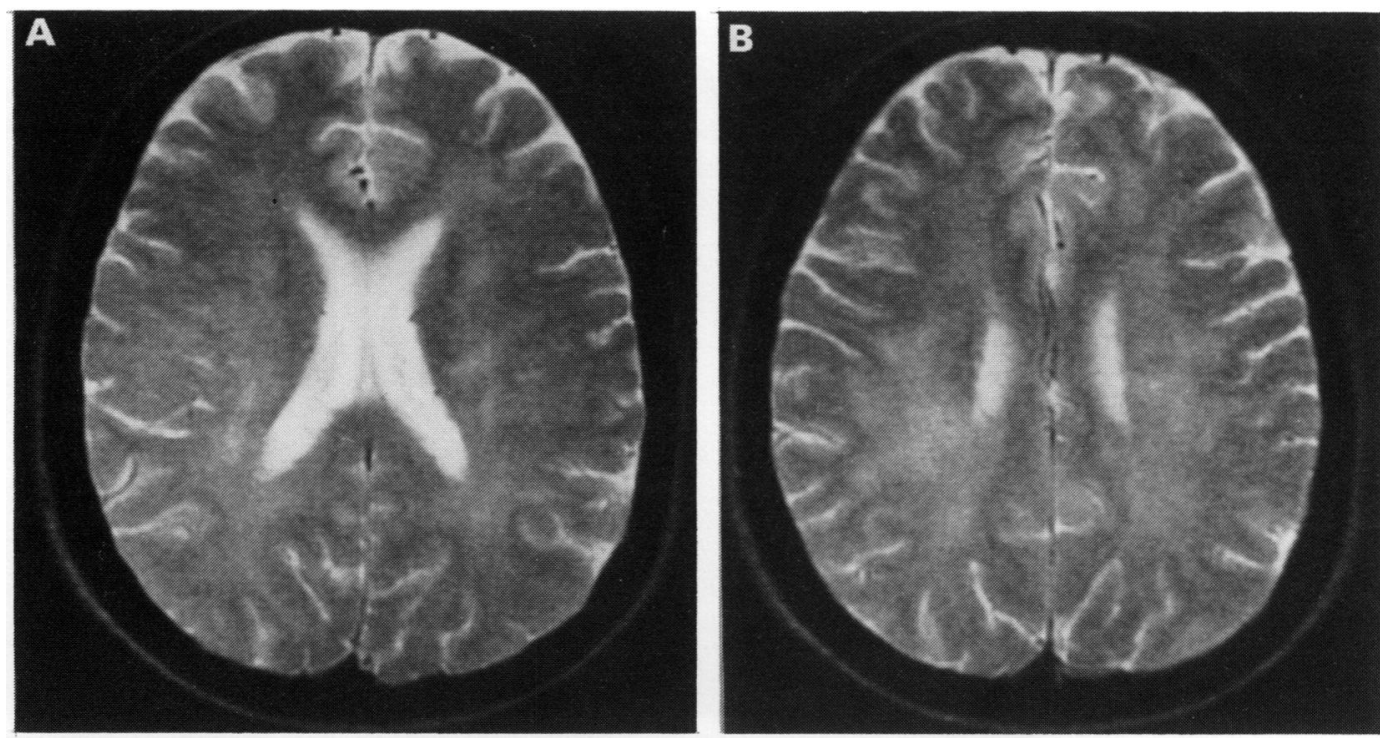

Figure 1 Axial $T_{2}$ weighted spin echo images from the March 1986 magnetic resonance imaging brain scan showing subtle confluent abnormally increased signal intensity affecting the parietal and occipital centrum semiovale and corona radiata bilaterally. Scan parameters: $1.5 \mathrm{~T}$; axial SE $5 \mathrm{~mm}$, TR $2500 \mathrm{~ms}, \mathrm{TE} 80 \mathrm{~ms}, 2 \mathrm{NEX}, 256 \times 256$ matrix.
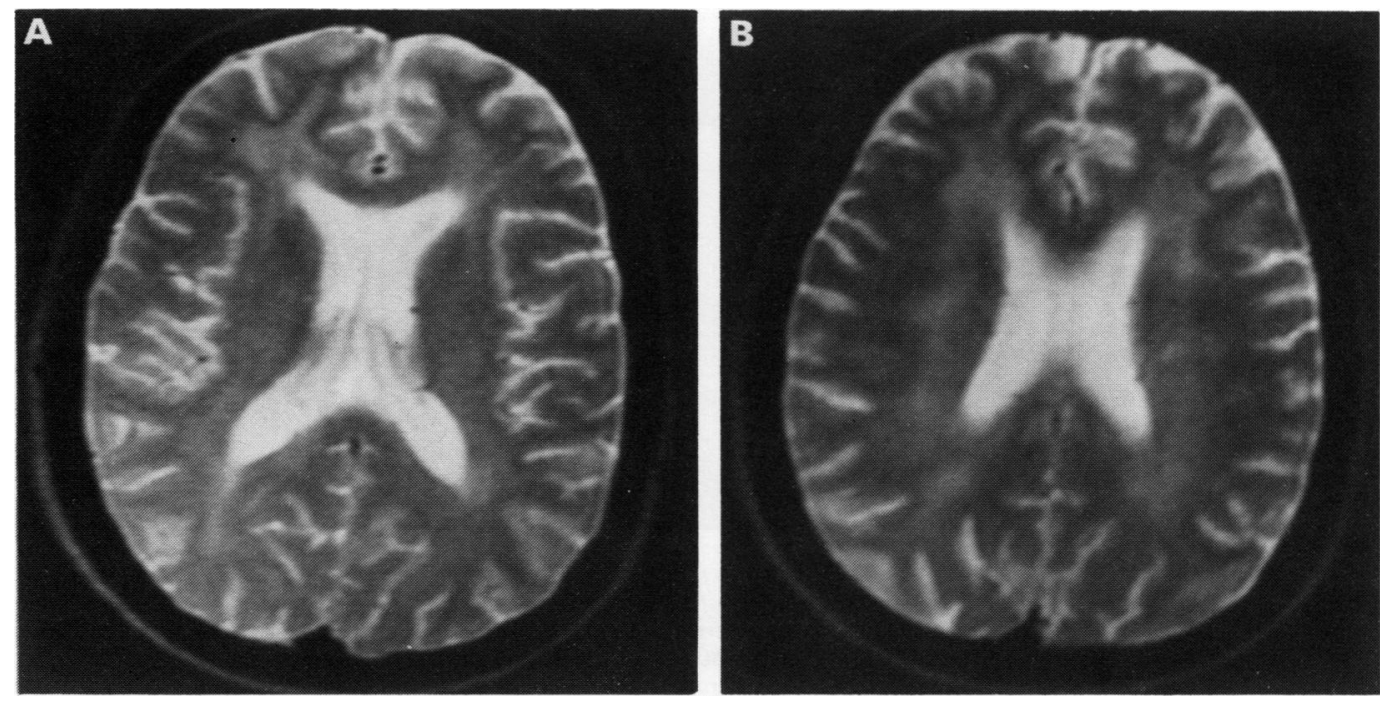

Figure 2 Axial $T_{2}$ weighted spin echo images from the $\mathcal{F}_{\text {une }} 1986$ magnetic resonance imaging brain scan showing progression of the abnormally increased signal intensity of the cerebral hemispheres which now affects the frontal lobes. There is also interval increase in the calibre of the lateral and third ventricles reflecting central cerebral atrophy. Scan parameters: $1.5 \mathrm{~T}$; axial SE $5 \mathrm{~mm}$, TR $2500 \mathrm{~ms}, \mathrm{TE} 80 \mathrm{~ms}, 2 \mathrm{NEX}$, matrix $256 \times 128$.

with lupus cerebritis. The dose of prednisone was increased and then gradually tapered over the next few months when her neurological and mental status improved.

In June 1986 she was again having difficulty concentrating and showed emotional lability. She had mild gait ataxia and a decrease in immediate recall of memory on examination. A three month follow up magnetic resonance imaging scan of the brain showed progressive demyelination predominately within the frontal centrum semiovale and coronal radiata. In addition the lateral and third ventricles showed striking enlargement, reflecting central cerebral atrophy (fig 2). An electroencephalogram performed in August 1986 showed a greater generalised non-specific abnormality than that performed in March 1986.

In October 1986 the patient became severely psychotically depressed and required admission to a hospital psychiatric ward. In addition she was forgetful and was having difficulty performing her job as a speech pathologist. She was leucopenic (white blood cell count $2.4 \times 10^{6} / 1$ ) and anaemic (packed cell volume 0.327 , direct Coombs' test negative). She received one dose of cyclophosphamide $(750 \mathrm{mg}$ ) intravenously and methylprednisolone $(100 \mathrm{mg})$ intravenously each day for three days. Her condition continued to deterioriate over the next few months.

In December 1986 she developed a shuffling gait, further difficulty with her memory, confusion, and increased emotional lability. She was disoriented with respect to time and had difficulty with simple calculations. She showed mild cogwheel rigidity and a subtle positional tremor. Cerebrospinal fluid analysis showed $0.005 \times 10^{6}$ white blood cells $/$, normal protein and glucose concentrations, and a negative Venereal Disease Research Laboratory (VDRL) and coccidioiodomycosis agglutination titre. The IgG synthesis rate was again increased at 

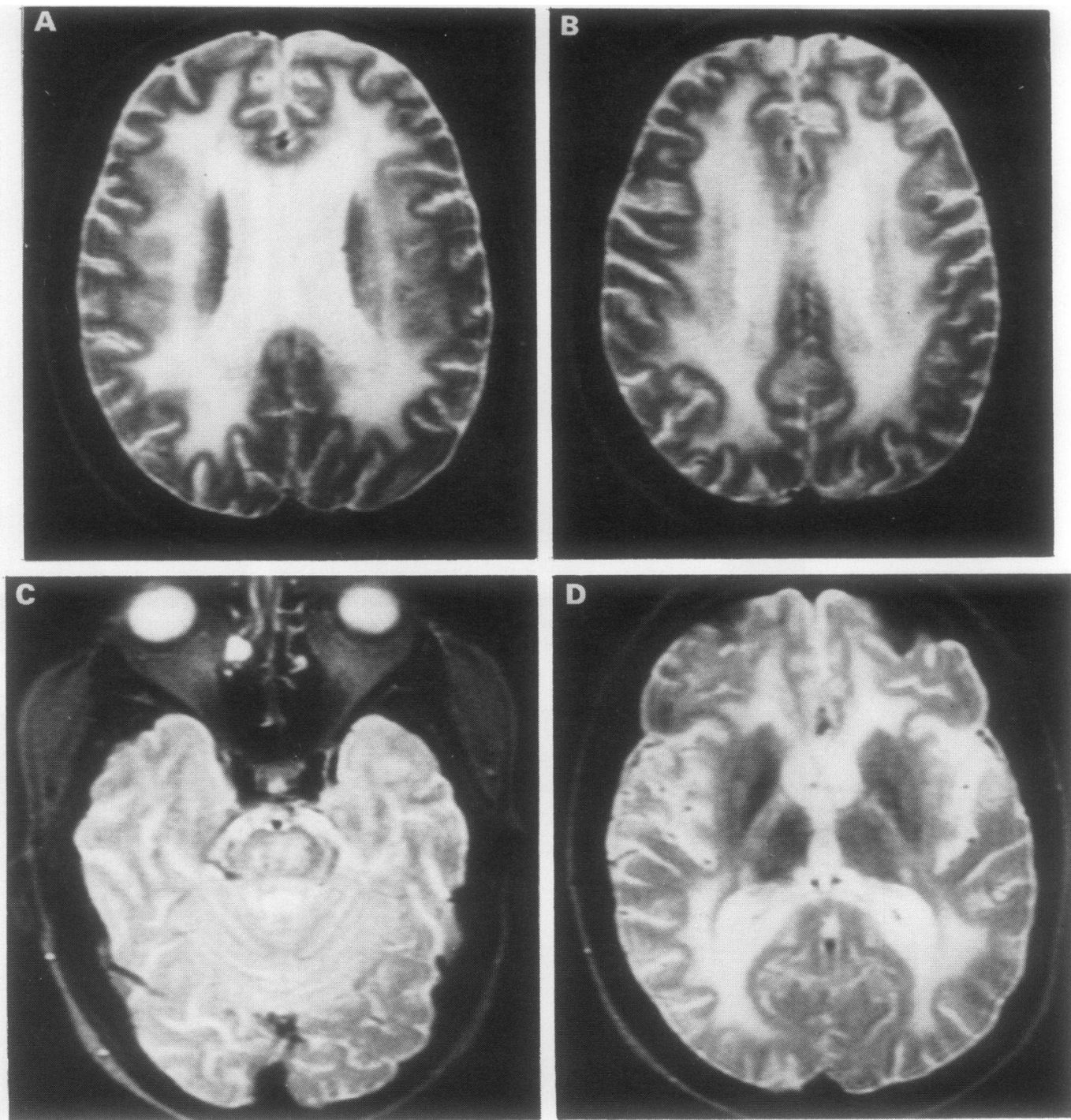

Figure $3 A$ and B: axial $T_{2}$ weighted spin echo images from the fourth magnetic resonance imaging brain scan (performed in December 1986) showing significant diffuse increase in demyelination throughout the cerebral hemispheres in addition to a significant increase in the ventricular size reflecting progressive central cerebral atrophy. Scan parameters: $1.5 T$; axial $S E 5 \mathrm{~mm}, T R 2500 \mathrm{~ms}, T E 80 \mathrm{~ms}$, matrix $256 \times 128,2 \mathrm{NEX}$. C and D: axial $T_{2}$ weighted spin echo images from the fourth magnetic resonance imaging brain scan (performed in December 1986) showing progressive demyelination affecting the posterior limbs of the internal capsules bilaterally as well as midbrain. Scan parameters: $1.5 T$; axial $S E 5 \mathrm{~mm}, T R 2500 \mathrm{~ms}$, TE $80 \mathrm{~ms}$, matrix $256 \times 128,2 \mathrm{NEX}$

$0.357 \mathrm{~g} / 24$ hours. Routine, mycobacterial, and fungal cultures of the cerebrospinal fluid were negative. Antibodies to double stranded DNA were slightly increased at $20 \%$ (Farr assay, normal less than 15\%). Total haemolytic complement was decreased at $48 \mathrm{U} / \mathrm{ml}$ (normal less than $64 \mathrm{U} / \mathrm{ml}$ ). She again received a single dose of cyclophosphamide (700 mg) intravenously, followed two weeks later by intravenous methylprednisolone $(250 \mathrm{mg}$ ) each day for three days.

A third magnetic resonance imaging scan of her brain showed an interval increase in the diffuse abnormality of the deep cerebral white matter in the cerebral hemispheres bilaterally, with no evidence of focal abnormalities nor masses. The scan also showed a progressive increase in the size of ventricles compared with the scan taken in June 1986. Two weeks later a fourth brain magnetic resonance imaging scan (fig 3A and B) showed a further progression of the diffuse white matter demyelination, which now affected the internal capsules bilaterally, mid pons, and both cerebral peduncles. The patient was then treated by plasmapheresis.
The rapid progression of the neurological disease raised the possibility that the patient might have PML, which cannot be treated. Consequently before using further intravenous cyclophosphamide and plasmapheresis to treat the patient's central nervous system lupus, a stereotactic needle biopsy sample was taken of the right frontal white cerebral matter at the University of California at San Francisco. The biopsy sample showed perivascular loss of myelin and reactive astrocytic gliosis (fig 4). No inflammatory infiltrates, microthrombi, nor vascular degeneration were present. There was no light nor electron microscopic evidence of PML. The findings most probably represent microischaemic lesions secondary to small vessel disease of the type associated with lupus cerebritis.

Despite a histological picture consistent with SLE, the patient's family decided to forego further treatment as she was moribund by this time. She was transferred to a nursing home and died in March 1987, almost five years after the onset of her SLE. A necropsy was not performed. 

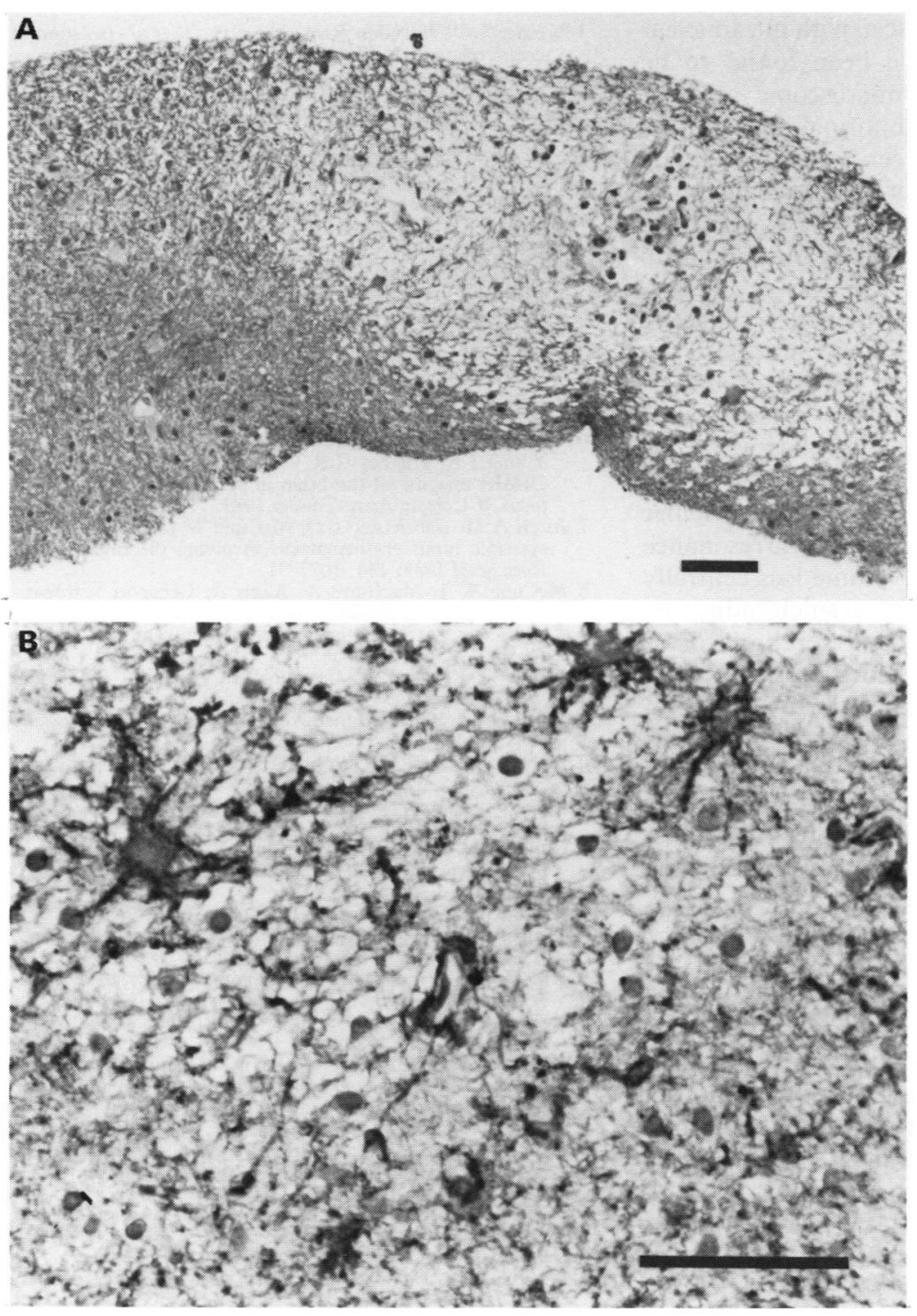

Figure 4 Needle biopsy sample of the frontal lobe white matter shows focal, perivascular loss of myelin with reactive astrocytic gliosis. (A) Luxol fast blue periodic acid-Schiff stain for myelin. (B) I mmunohistochemistry for glial fibrillary acidic protein with haematoxylin counterstain shows large reactive astrocytes and their processes surrounding a centrally placed blood vessel. Macrophages with clear cytoplasm are also present in this field. Scale bars are $50 \mu \mathrm{m}$. performed. Our patient eventually developed extremely abnormal magnetic resonance imaging scans of the brain. Brain magnetic resonance imaging scans can be useful in assessing the activity of central nervous system lupus. $^{6-10}$ Generally the magnetic resonance imaging scan shows more focal lesions than can be seen with computer assisted tomography. Cerebral infarcts appear as a relatively large area of increased signal on proton density and $T_{2}$ weighted images. Microinfarcts or ischaemic demyelination may cause multiple small areas of increased signal intensity in the white matter. $^{7910}$ Focal areas of increased signal intensity have also been noted in the central grey area. These abnormalities may resolve with treatment. ${ }^{7}$ Cerebral atrophy also commonly occurs. $^{8}$ Clinical improvement in central nervous system lupus has been noted to correlate with magnetic resonance imaging findings. ${ }^{79}$ Interestingly, Lim et al found no correlation with SLE psychiatric disease and magnetic resonance imaging findings. ${ }^{15}$ The findings on magnetic resonance imaging scans of the brain in patients with central nervous system lupus are non-specific. Similar changes can be seen in patients with demyelinating or inflammatory conditions such as multiple sclerosis, atherosclerotic microangiopathic disease, HIV encephalitis, and other vasculidities.

Evidence of old central nervous system disease may not resolve on the magnetic resonance imaging scan. Consequently adjunctive tests such as the IgG synthesis rate can be helpful in assessing current central nervous system lupus activity. IgG synthesis rates are thought to reflect the intrathecal formation of immunoglobulins. ${ }^{16}$ Several studies have shown a good correlation between IgG synthesis rate or index, or both, and central nervous system activity. ${ }^{17-19}$ This correlation was certainly present in our patient who had markedly increased IgG synthesis rates while her central nervous system disease was active.

Central nervous system manifestations of SLE generally occur early in the course of the disease, usually within the first 12 months. ${ }^{20}$ Our patient showed symptoms of SLE for almost three years before developing neuropsychiatric manifestations, showing that central nervous system lupus can occur at any time.

A review of published work shows six cases of PML reported in patients with SLE. ${ }^{15}$ All of these patients had been receiving immunosuppressive drugs such as corticosteroids or azathioprine, or both. Progressive multifocal leucoencephalopathy is thought to be caused by papovaviruses $^{21} 22$ and is associated with immunosuppressed states such as haematological malignancies, especially chronic lymphocytic leukaemia and lymphoma, other malignancies, and in patients receiving long term corticosteroid treatment or who are otherwise immunosuppressed. ${ }^{5}$ 23 It has also been reported to occur in patients with acquired immunodeficiency syndrome (AIDS). ${ }^{23}$ The usual clinical presentation of PML is of multiple neurological abnormalities which progress rapidly. ${ }^{24}$ Classically the diagnosis of $P M L$ is made at necropsy when extensive axonal and malities, as our patient illustrates well. As there is no single definitive test for diagnosing and evaluating the activity of central nervous system lupus, a battery of tests generally needs to be 
neuronal demyelination is seen with intranuclear inclusion bodies that have been found to be papovavirus on electron microscopic study. ${ }^{25}$ Our patient's diffuse abnormalities of the cerebral white matter seen on a magnetic resonance imaging scan of the brain and her great variety of unusual, rapidly progressing neurological symptoms suggested the diagnostic possibility of PML.

The classic magnetic resonance imaging findings in PML include lesions limited to the white matter of the brain that are often round or oval and then become confluent. These lesions can be fairly large. Usually no mass effect is seen. The white matter disease is often asymmetric and distant from the periventricular region. ${ }^{11}$ Our patient's brain magnetic resonance imaging scan also showed volume loss centrally with preservation of the cortical sulci, indicating preservation of the peripheral cerebral cortex and involution of central white matter. Radiographically these findings indicate a microangiopathic process. These features suggested that the magnetic resonance imaging scan findings in our patient were more likely to be due to central nervous system lupus than PML. The decision to continue aggressively treating the patient for central nervous system lupus, however, required a pathological differentiation of the two diseases.

We had the rare opportunity to correlate brain magnetic resonance imaging scan findings and pathology while the patient was still alive. Our patient's cerebral biopsy sample showed reactive astrocytic gliosis and non-specific white matter degeneration, and none of the classic histological findings of PML such as perivascular inflammation, bizarre reactive astrocytes, or intranuclear viral inclusions. The pathological findings in central nervous system lupus, on the other hand, are often protean, and may be nonspecific. The more common central nervous system pathological findings in SLE include microinfarcts, perivascular infiltrates, and fibrinoid degeneration in the vessel walls. ${ }^{1626}$ True arteritis is rare in central nervous system lupus. ${ }^{1626}$

In summary our case illustrates the problems with some of the diagnostic tests in central nervous system lupus and the need to carry out several diagnostic procedures in evaluating central nervous system SLE, and to be aware of the difficulties in differentiating PML from central nervous system lupus in certain patients. Furthermore florid neuropsychiatric lupus with rapid white matter changes could clinically be confused with an unusual infectious process such as PML. With advanced technology we can sometimes make a distinction allowing earlier diagnosis and treatment.

The authors thank Dr Ronald L Kaye, Dr Kenneth Sack, and Ms Fran Tannenbaum Kaye for their helpful comments, and Ms Nina August for her assistance in typing the manuscript.
1 Weiner L P, Herndon R M, Narayan O, et al. Isolation of virus related to SV40 from patients with progressive virus related to SV40 from patients with progressive
multifocal leukoencephalopathy. $N$ Engl f Med 1972; 286: multifocal

2 Malas D, Weiss S. Progressive multifocal leukoencephalopathy and cryptococcal meningitis with systemic lupus erythematosus and thymoma. Ann Neurol 1977; 1: 188-91.

3 Ellis S G, Verity M A. Central nervous system involvement in systemic lupus erythematosus: a review of neuropathologic findings in 57 cases, 1955-1977. Semin Arthritis Rheum 1979; 8: 212-21

4 Jones H R Jr, Hedley-Whyte E T, Freidberg S R, Kelleher J E Jr, Krolikowski J. Primary cerebellopontine progressive cerebellar biopsy. Ann Neurol 1982; 11: 199-202.

5 Newton P, Aldridge $R$ D, Lessells A M, Best P V. Progressive multifocal leukoencephalopathy complicating Progressive multifocal leukoencephalopathy complicating
systemic lupus erythematosus. Arthritis Rheum 1986; 29: systemic

6 Vermess M, Bernstein R M, Bydder G M, Steiner R E, Young I R, Hughes $G \mathbf{R}$ V. Nuclear magnetic resonance (NMR) imaging of the brain in systemic lupus erythematosus. F Comput Assist Tomogr 1983; 7: 461-7

7 Aisen A M, Gabrielsen T O, McCune W J. MR imaging of systemic lupus erythematosus involving the brain. $A m \mathcal{F}$ Roentgenol 1985; 144: 1027-31.

8 McCune W J, MacGuire A, Aisen A, Gebarski S. Identification of brain lesions in neuropsychiatric systemic lupus erythematosus by magnetic resonance scanning. Arthritis Rheum 1988; 31: 159-66.

9 Jacobs L, Kinkel P R, Costello P B, Alukal M K, Kinkel W R, Green F A. Central nervous system lupus erythematosus: the value of magnetic resonance imaging. $\mathcal{f}$ Rheumatol 1988; 15: 601-6.

10 Bell C L, Partington K, Robbins M, Graziano F M, Turski P Kornguth S E. Cranial MRI scans in patients with central
Kat Kornguth S E. Cranial MRI scans in patients with central
nervous system (SLE) lupus. Arthritis Rheum 1989; 32 nervous system

11 Guilleux $M \mathrm{H}$, Steiner R E, Young I R. MR imaging in progressive multifocal leukoencephalopathy. AfNR 1986; 7: $1033-5$.

12 Carbotte R M, Denburg S D, Denburg J A. Prevalence of cognitive impairment in systemic lupus erythematosus. f Nerv Ment Dis 1986; 174: 357-64.

13 Kremer J M, Rynes R I, Bartholomew L E, et al. Nonorganic non-psychotic psychopathology (NONPP) in patients with systemic lupus erythematosus. Semin Arthritis Rheum 1981; 11: 182-9.

14 Devinski O, Petito C K, Alonso D R. Clinical and neuropathological findings in systemic lupus erythematosus: the pathological findings in systemic lupus erythematosus: the
role of vasculitis, heart emboli, and thrombotic thromborole of vasculitis, heart emboli, and thrombotic thro
cytopenic purpura. Ann Neurol 1988; 23: 380-4.

15 Lim L, Ron M A, Ormerod I E C, et al. Psychiatric and neurological manifestations in systemic lupus erythematosus. Qf Med 1988; 66: 27-38.

16 Bluestein H G. Neuropsychiatric disorders in systemic lupus erythematosus. In: Lahita R G, ed. Systemic lupus erythematosus. New York: Wiley, 1987: 593-614.

17 Winfield J B, Shaw M, Silverman L M, Eisenberg R A Wilson H A III, Koffler D. Intrathecal IgG synthesis and blood-brain barrier impairment in patients with systemic lupus erythematosus and central nervous system dysfunction. Am $\mathcal{F}$ Med 1983; 74: 837-44.

18 Hirohata S, Hirose S, Miyamoto T. Cerebrospinal fluid IgM, IgA, and IgG indexes in systemic lupus erythematosus: their use as estimates of central nervous system disease activity. Arch Intern Med 1985; 145: 1843-6.

19 Ernerudh J, Olsson T, Lindstrom F, Skogh T. Cerebrospinal fluid immunoglobulin abnormalities in systemic lupus erythematosus. F Neurol Neurosurg Psychiatry 1985; 48: erythem $807-13$.

20 Feinglass E J, Arnett F C, Dorsch C A, Zizic T M, Stevens $M$ B. Neuropsychiatric manifestations of systemic lupus erythematosus: diagnosis, clinical spectrum, and relationship to other features of the disease. Medicine (Baltimore) 1976; 55: 323-9.

21 Woodhouse N A, Dayan A D, Burston J. Progressive multifocal leukoencephalopathy: electron microscopic study of four cases. Brain 1967; 90: 863-70.

22 Padgett B L, Walker D L, Zu Rheim G M, Eckroade R J, Dessel B H. Cultivation of papova-like virus from human brain with progressive multifocal leukoencephalopathy. Lancet 1971; i: $1257-60$.

23 Krupp L B, Lipton R B, Swerdlow M L, Leeds N E, Llena J Progressive multifocal leukoencephalopathy: clinical and Progressive multifocal leukoencephalopathy: clinical
radiographic features. Ann Neurol 1985; 17: 344-9.

24 Richardson E P Jr. Progressive multifocal leukoencephalopathy. N Engl Jr. Med 1961; 265: 815-23.

pathy. N Engl F Med 1961; 265: 815-23.
25 Richardson E P, Webster H. Progressive multifocal leukoencephalopathy: its pathological features. Prog Clin Biol Res 1983; 105: 183-90.

26 Johnson R T, Richardson E P. The neurological manifestations of systemic lupus erythematosus: a clinical-pathological study of 24 cases and review of the literature. Medicine (Baltimore) 1968; 47: 337-69. 